\title{
Plastic and Reconstructive Surgery
}

\section{CHRONIC SWELLING OF THE LEG AND STASIS ULCER}

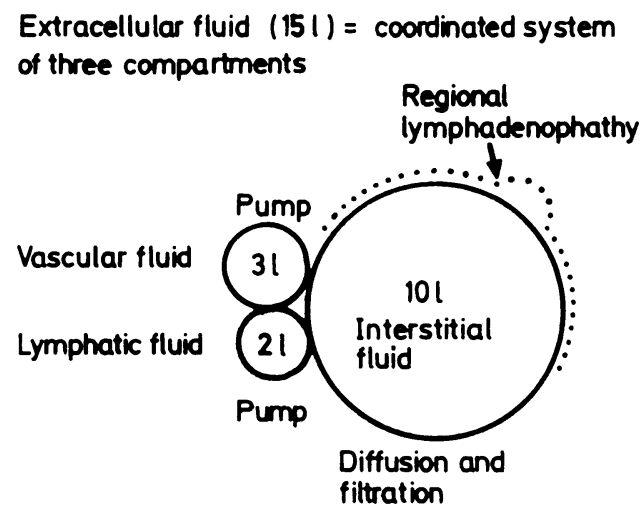

\section{Oedema of venous origin}

\begin{tabular}{|c|c|c|c|}
\hline \multicolumn{4}{|c|}{ Clinical classitication of varicose veins } \\
\hline \multirow[t]{2}{*}{ Type } & \multicolumn{3}{|c|}{ Veins } \\
\hline & Epitascial & Perforating & Deep \\
\hline I Asymptomatic & $\underset{\substack{\text { Abnormal } \\
(+1}}{ }$ & Normal & Normal \\
\hline II Symptomatic & $\underset{(++)}{\text { Abnormal }}$ & $\begin{array}{l}\text { Abnormal } \\
(+1\end{array}$ & Normal \\
\hline \multicolumn{4}{|l|}{$\begin{array}{l}\text { III With trophic } \\
\text { change: }\end{array}$} \\
\hline Epifascial & $\underset{(+++)}{\text { Abnormal }}$ & $\underset{(+++)}{\text { Abnormal }}$ & Normal \\
\hline Subtascial & Abnormal & $\underset{(+++1}{\text { Abnormal }}$ & $\begin{array}{c}\text { Abnormal } \\
(+/+++1\end{array}$ \\
\hline
\end{tabular}

Swelling of the leg is a condition often seen in general practice. Most commonly it develops secondarily to a local abnormality in the venous or lymphatic system, but systemic abnormalities such as cardiac or renal failure or myxoedema should not be overlooked.

By definition oedema is a pathological accumulation of fluid in the interstitial tissues. Physiologists divide the body water into intracellular and extracellular compartments, extracellular compartments consisting of the vascular circulation, lymphatic circulation, and interstitial "fluid." The interstitial fluid is a thin layer of gel, a colloidal system in which particles of solid are dispersed in a liquid. The interstitial compartment has a capacity to imbibe water but any such tendency is opposed by the osmotic pressure of the plasma, drawing water into the capillaries. The viability of cells is maintained by the continuously occurring transit of plasma fluid into the interstitial spaces (outward filtration) and back again (inward filtration). This transcapillary fluid exchange is in perfect balance under normal conditions. If this balance is disturbed the sol phase of the colloid increases its volume and oedema arises.

More than $90 \%$ of swollen legs seen in general practice result from incompetence of the venous system. This reversible orthostatic oedema (oedema arising after standing and disappearing when the venous perfusion pressure is lowered by raising the foot) is considered to be a dominating sign of impaired venous drainage of the leg. The terms varicose veins and epifascial chronic venous insufficiency are used when the pathology is limited to the superficial veins alone and the post thrombotic syndrome and deep chronic venous insufficiency when the oedema is induced by thrombosis of the deep veins.

Three clinical groups of patients with chronic varicose veins can be identified according to the severity of their signs and symptoms. 

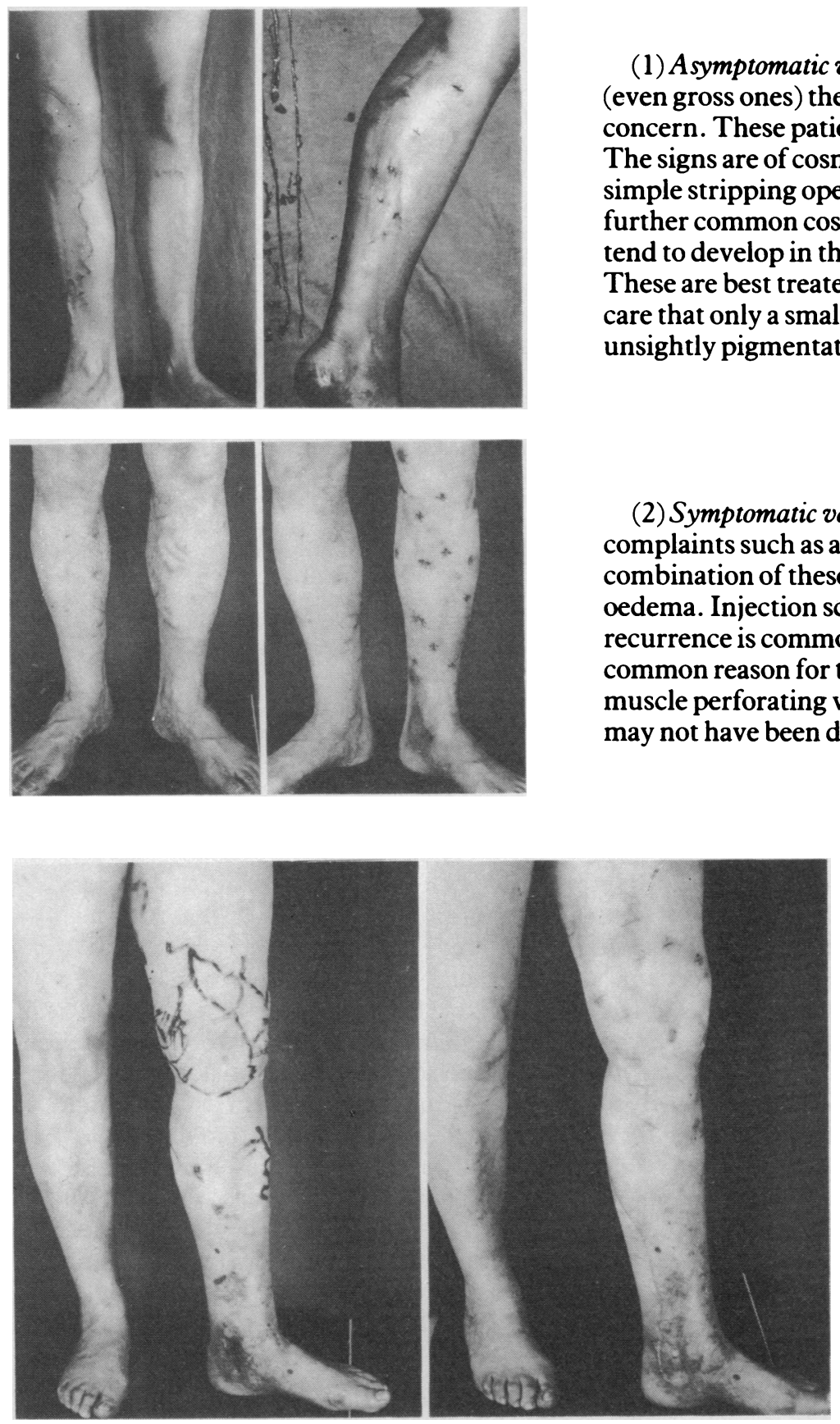

\section{Oedema of lymphatic origin}

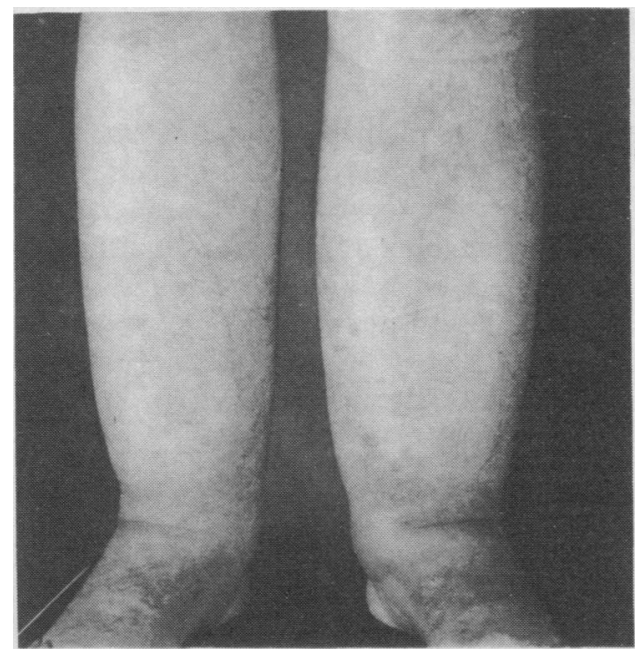

(1) Asymptomatic varicose veins - In most patients with varicose veins (even gross ones) the orthostatic oedema is so mild that it is of little clinical concern. These patients represent a well defined and easily treated group. The signs are of cosmetic relevance alone, and the therapeutic results after a simple stripping operation or injection of a sclerosant are excellent. A further common cosmetic complaint is the presence of spider veins, which tend to develop in the upper thigh and are very fine cutaneous venules. These are best treated by microinjections using a $26 \mathrm{G}$ needle and taking care that only a small amount of weak sclerosant is used; otherwise unsightly pigmentation may occur.

(2) Symptomatic varicose veins - The second group includes patients with complaints such as a feeling of heaviness, itching, tension, or cramps, or a combination of these, in the affected leg together with visible orthostatic oedema. Injection sclerotherapy usually gives poor long term results, and recurrence is common after the conventional stripping operation. The most common reason for this is the presence of several pathologically dilated muscle perforating veins or of an incompetent short saphenous vein that may not have been dealt with adequately at the first operation.

(3) Varicose veins with trophic changes - These patients display the highest morbidity. Their condition is characterised by the presence of trophic changes ranging from induration of the subcutaneous tissue to pigmentation, skin atrophy, and ulceration. Usual but not obligatory is impairment of both the deep and superficial venous drainage systems. Irrespective of the aetiology, the most important pathological factor is, in addition to epifascial varicosities, a vastly increased number of incompetent transfascial communicating veins resulting in severe dysfunction of the foot and calf muscle pumps. The swollen leg with extensive trophic changes responds poorly to compression treatment as well as to injection sclerotherapy and often recurs after conventional stripping operations. Satisfactory long term results can best be achieved from a dorsal subfascial approach, raising a long medial and lateral fasciocutaneous flap and ligating all dilated incompetent perforating veins.

This form of swelling results from impaired transport of lymph, which arises either spontaneously (primary lymphoedema) or secondarily to damage of the lymphatic system, most often in this country after surgical exenteration of the regional lymph nodes (secondary lymphoedema). Lymphoedema is typically unilateral but if it is bilateral it is always asymmetrical and has from the beginning little tendency to reversibility: when the patient gets up in the morning the leg is still swollen. 

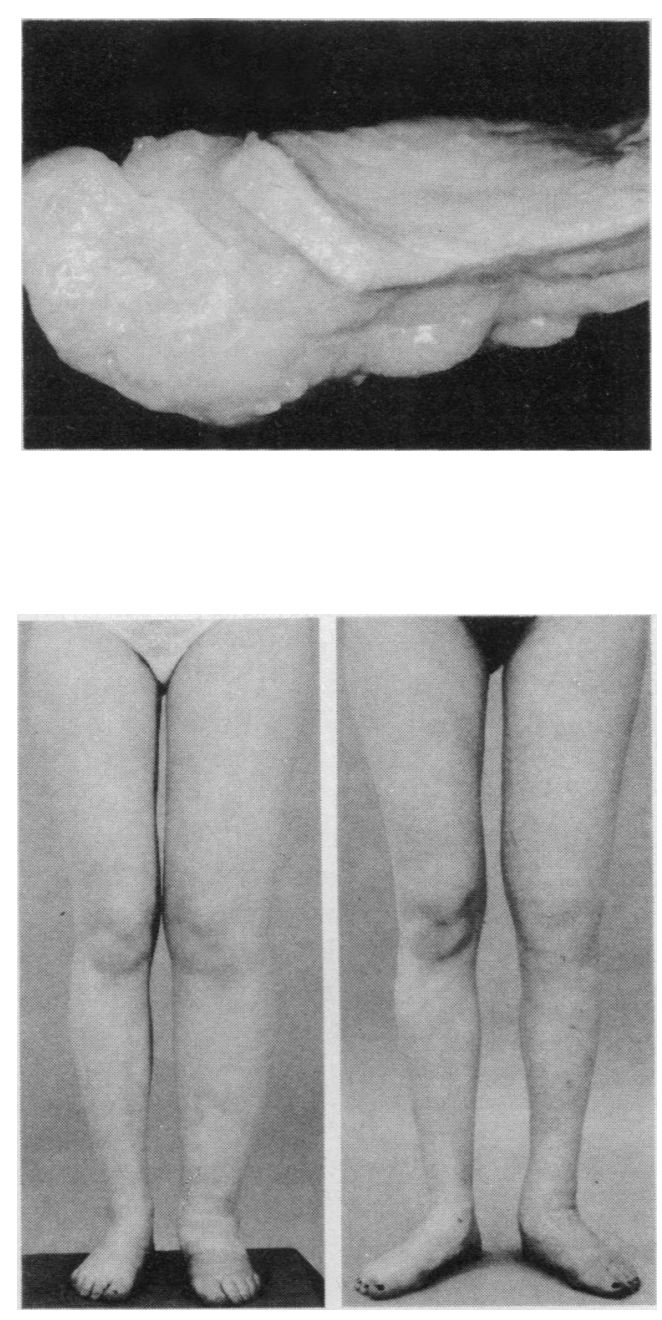

\section{Lipoedema}

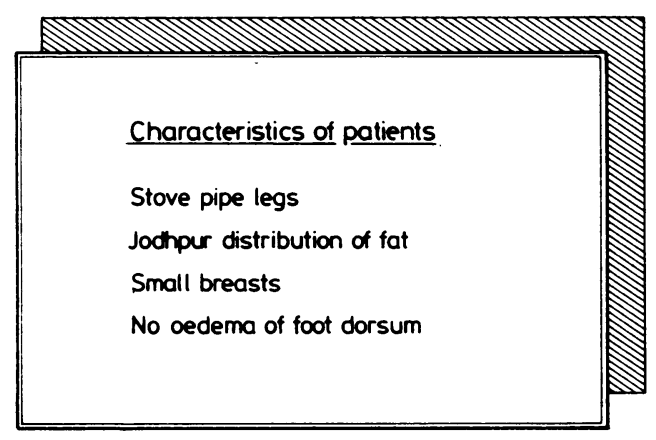

\section{Stasis ulcers}

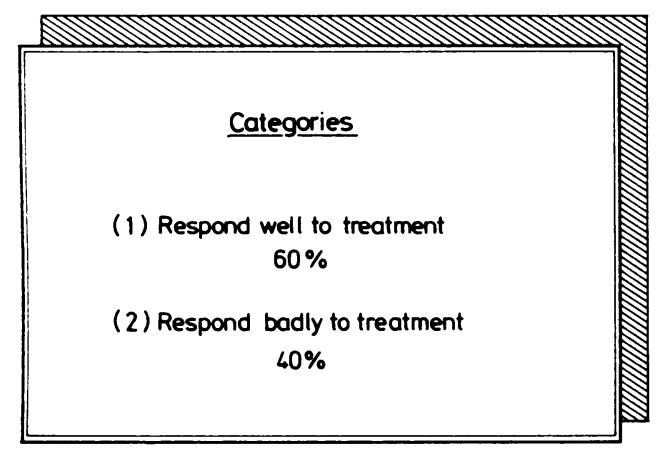

Lymphoedema, like venous oedema, is due to the overaccumulation of fluid in the interstitial spaces, but, whereas in venous oedema this is due to a pathological increase of intracapillary pressure, in lymphoedema it is related to an increased protein content in the interstitial fluid. In contrast to chronic venous insufficiency, the morphological changes induced by lymphatic impairment usually result in progressive increase in the girth of the affected extremity without clinically evident damage to the skin. The trophic changes, consisting mainly in severe fibrosis of the subcutaneous tissue and thickening of the fascia, have two important therapeutic consequences:

(1) The effect of externally applied pressure to the subcutis is considerably reduced (in severe fibrosis only $10 \%$ of the externally applied pressure may reach the subcutis).

(2) Compression treatment can effectively treat the overaccumulation of water but cannot reduce the enlarged girth due to fibrosis.

Nevertheless, the basic treatment for lymphoedema is compression treatment. It consists of the combined application of external intermittent compression by an electric pump and continuous compression by an elastic stocking of adequate compression grade. In advanced forms of lymphoedema with severe fibrosis of the skin and subcutaneous tissue the only effective treatment to reduce the girth is surgery. The operations performed in the past 80 years have aimed at either the improvement of lymph flow-drainage operations-or the reduction of the girthexcisional operations. In our hands all drainage operations have turned out to be unsatisfactory. The only operation consistently giving good long term results in both primary and secondary lymphoedema is the staged resection of the subcutaneous tissue along with thickened fascia and preservation of skin in the form of thin flaps. The operation improves the appearance of the leg, maintains the massage effect of the underlying muscles on the skin and makes the patient responsive to less vigorous compression treatment.

Lipoedema, or lipidosis, occurs predominantly in young women and is characterised by bilateral moderate enlargment of the legs, without oedema, in the presence of normal veins and normal or minimally hypoplastic lymph ducts. Characteristically the patient has small breasts, a jodhpur distribution of fat in the thighs, large legs (stove pipe leg), and no oedema of the dorsum of the foot. The only available treatment that can be recommended is liposuction of the fat accumulation in the thighs.
Most chronic ulcers of the leg are of venous origin. The immediate cause is a break down of the microcirculation with inadequate oxygenation of the affected area of skin. The typical site of a venous ulcer is above the medial malleolus, and, according to the "blow out" concept, they result from the incompetence of a supramalleolar ankle perforating vein. Many patients, however, with grossly dilated and incompetent ankle perforating veins have no trophic changes of the gaiter area whatsoever. In our experience a simultaneous dilatation and incompetence of all the main longitudinal veins along with most of the perforating veins is necessary for the development of an ulcer and its persistence. 


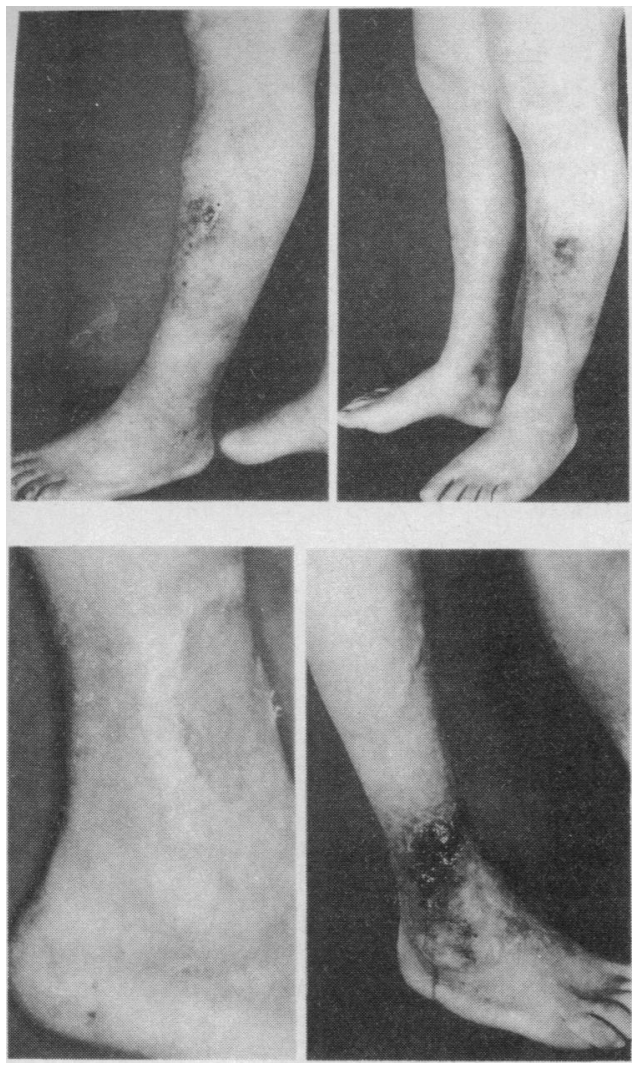

With regard to treatment chronic venous ulcers may be divided into two categories: those $(60 \%)$ that respond well to treatment and those $(40 \%)$ that do not. In those that respond well the ulcer develops above a cushion of dilated veins one of which is usually but not necessarily a perforating vein. Once these veins have been eliminated by compression, injection, or surgery the ulcer heals spontaneously or more rapidly with a partial thickness skin graft. This skin graft should remain supported by adequate elasticated compression from the toes to the knee at all times when the leg is dependent postoperatively and should be continued indefinitely.

In ulcers that do not respond well the dependence on dilated veins is not evident and the main pathophysiological factor is severe dysfunction of the calf muscle pump. This in turn results from several factors occurring in various combinations. They are: an increased number of incompetent communicating veins; varicosities of both long and short saphenous veins; a restricted range of movement at the ankle and metatarsal joints; and a "chronic compartment syndrome" due to the induration of epifascial tissues. The treatment of choice is the improvement of the muscle pump effect by a subfascial operation ligating incompetent perforating veins combined with skin grafting of the ulcer.

Less often the cause of non-healing is mycotic infection, a lowered resistance to infection by the patient, or arterial insufficiency. More rarely ulcers may be of tuberculous or syphilitic origin or a complication of systemic diseases such as ulcerative colitis. It should also be borne in mind that malignancy can occur in long standing ulcers and that a biopsy should therefore be performed whenever there is doubt about the diagnosis.

Dr J J Pflug, MD, PHD, is honorary consultant and senior lecturer, Royal Postgraduate Medical School, Hammersmith Hospital, and Mr D M Davies, FRCS, is consultant plastic surgeon, West London Plastic Surgery Centre, West Middlesex University Hospital, Isleworth, and consultant plastic surgeon and honorary senior lecturer, Royal Postgraduate Medical School, Hammersmith Hospital, London W12 0HS.

\title{
Impressions of Medicine in India
}

\section{A doctor's lot}

\author{
TESSA RICHARDS
}

Medicine is an attractive career in India; a doctor has the potential to earn a good salary and the profession is generally held in high esteem. And, unlike Britain, where exhaustive media coverage of matters medical has resulted in an aware and at times demanding public, medicine in India remains mysterious - which if not necessarily in the patients' best interests is often in the best interest of the profession. But all is not a bed of roses, and as the pool of trained doctors grows and as it becomes harder to find work in former outlets such as America and England competition in the urban areas (and few want to go to a rural area) is intense and unemployment a reality. Furthermore, the system of job allocation and promotion is such that career prospects (irrespective of merit) are a good deal more equal for some than for others.

British Medical Journal, London WC1H 9JR

TESSA RICHARDS, MRCP, MRCGP, assistant editor

\section{Getting a foothold}

The road ahead is tough for newly qualified graduates. If they fail to gain a place on a postgraduate training rotation or an appointment in the state government or central government services-and only a minority do-they must either opt for hospital attachments, jobs for which they actually have to pay a monthly stipend, or try to find a niche in the private sector. For many this means a job as a house officer in either a large private hospital or a small private nursing home or clinic. I talked to one young doctor in his room in one of the larger private hospitals in Bombay. The room was small and shabby, not dissimilar to the average on call room in Britain, but he had to share it with two other doctors. Furthermore, he had slept in it every night over the last three weeks because he had no official time off, cover being arranged on an ad hoc basis. His pay was half that for the equivalent job in a state hospital, which made it less than that of the sweepers and liftmen. But the poor salary and accommodation, and long working hours would be acceptable (for in this respect junior jobs in the private sector are not that much worse than state hospital posts) if the posts were "recognised" to give suitable 